OPEN ACCESS

Edited by:

Jacqueline Batley,

The University of Western Australia,

Australia

Reviewed by:

Martin Mascher,

Leibniz-Institut für Pflanzengenetik und Kulturpflanzenforschung (IPK),

Germany

Gunvant Baliram Patil,

University of Minnesota, United States

${ }^{*}$ Correspondence:

Fatemeh Maghuly

fatemeh.maghuly@boku.ac.at

Margit Laimer

margit.laimer@boku.ac.at

Specialty section: This article was submitted to Plant Breeding,

a section of the journal

Frontiers in Plant Science

Received: 11 January 2018

Accepted: 04 April 2018

Published: 23 April 2018

Citation:

Maghuly F, Pabinger S, Krainer $J$ and

Laimer M (2018) The Pattern and

Distribution of Induced Mutations in

J. curcas Using Reduced

Representation Sequencing.

Front. Plant Sci. 9:524.

doi: $10.3389 / f p / s .2018 .00524$

\section{The Pattern and Distribution of} Induced Mutations in $J$. curcas Using Reduced Representation Sequencing

\author{
Fatemeh Maghuly ${ }^{1 *}$, Stephan Pabinger ${ }^{2}$, Julie Krainer ${ }^{2}$ and Margit Laimer ${ }^{1 *}$ \\ ${ }^{1}$ Plant Biotechnology Unit, Department of Biotechnology, Vienna Institute of BioTechnology, University of Natural Resources \\ and Life Sciences, Vienna, Austria, ${ }^{2}$ Molecular Diagnostics, Center for Health \& Bioresources, Austrian Institute of \\ Technology, Vienna, Austria
}

Mutagenesis in combination with Genotyping by Sequencing (GBS) is a powerful tool for introducing variation, studying gene function and identifying causal mutations underlying phenotypes of interest in crop plant genomes. About 400 million paired-end reads were obtained from 82 ethylmethane sulfonate (EMS) induced mutants and 14 wild-type accessions of Jatropha curcas for the detection of Single Nucleotide Polymorphisms (SNPs) and Insertion/Deletions (InDels) by two different approaches (nGBS and ddGBS) on an Illumina HiSeq 2000 sequencer. Using bioinformatics analyses, 1,452 induced SNPs and InDels were identified in coding regions, which were distributed across 995 genes. The predominantly observed mutations were G/C to A/T transitions (64\%), while transversions were observed at a lower frequency (36\%). Regarding the effect of mutations on gene function, $18 \%$ of the mutations were located in intergenic regions. In fact, mutants with the highest number of heterozygous SNPs were found in samples treated with $0.8 \%$ EMS for $3 \mathrm{~h}$. Reconstruction of the metabolic pathways showed that in total 16 SNPs were located in six KEGG pathways by nGBS and two pathways by ddGBS. The most highly represented pathways were ether-lipid metabolism and glycerophospholipid metabolism, followed by starch and sucrose metabolism by nGBS and triterpenoid biosynthesis as well as steroid biosynthesis by ddGBS. Furthermore, high genome methylation was observed in J. curcas, which might help to understand the plasticity of the Jatropha genome in response to environmental factors. At last, the results showed that continuously vegetatively propagated tissue is a fast, efficient and accurate method to dissolve chimeras, especially for long-lived plants like J. curcas. Obtained data showed that allelic variations and in silico analyses of gene functions (gene function prediction), which control important traits, could be identified in mutant populations using nGBS and ddGBS. However, the handling of GBS data is more difficult and more challenging than the traditional TILLING strategy in mutated plants, since the Jatropha genome sequence is incomplete, which makes alignment and variant analysis of target sequence reads challenging to perform and interpret. Therefore, providing a complete Jatropha reference genome sequence with high quality should be a priority for any breeding program.

Keywords: chemical mutagenesis, SNP calling, crop improvement, biofuel, reverse genetic 


\section{INTRODUCTION}

Jatropha curcas (family Euphorbiaceae) originates from Central America and currently is distributed throughout all tropical and subtropical regions. It could be one of the most valuable crops, not only as a biofuel plant but also due to the high protein amount in its seeds, which make it attractive as animal feed and organic manure. Beside its variable toxin content, consisting mainly of diterpenes (phorbol esters) and ribosomeinactivating proteins (curcin) (Maghuly et al., 2013, 2016), the plant raises interest for its ability to produce pharmaceutically important compounds. However, the genome of J. curcas is highly homogenous $(C=0.416 \times 109 \mathrm{bp}, 2 \mathrm{n}=22)$, and its genetic diversity is low (Carvalho et al., 2008; Maghuly et al., 2015; Maghuly and Laimer, 2017). Compared to other oilseed crops, research and development of $J$. curcas is still at a very early stage (King et al., 2013), however, the release of the whole genome sequence of J. curcas $^{1}$ opened a new era in genomic research (Sato et al., 2011; Zhang et al., 2014; Wu et al., 2015). Nevertheless, data from genome projects alone will not automatically offer solutions for increasing productivity, decreasing toxin amounts and detailed understanding of pathogen resistance. Still, detailed information of the genetic landscape and gene function is a premise to (a) uncover why some accessions of $J$. curcas produce toxins and others not, (b) why - with their narrow genetic variation - J. curcas can grow in such diverse environmental conditions, (c) know, how biochemistry contributes to increases in oil yield and quality, (d) understand the processes of growth and development.

Induced mutations are useful to study gene functions in populations with a uniform genetic background, like J. curcas, as their genetic variation is typically dissolved (Henry et al., 2014). Further, chemical and physical mutagenesis has a long history for plant breeding to identify and select desirable traits (e.g., oil quality and low toxicity) for crop improvement, and is shown to be an effective method to induced mutagenesis in a wide range of plants (Bado et al., 2015). These methods provide necessary population resources, structural variation, and the identification of gene functions by gene knockouts, disruptions, and missense. Physical mutagenesis could produce huge DNA alterations such as large deletions, from 1 to $230 \mathrm{kbp}$, with frame-shifts or total gene loss, inversions, translocations and small intragenic mutations, while chemical mutagenesis mainly causes point mutations (base substitutions), small insertions and deletions (Mba et al., 2010). Compared to various type of chemical mutagenesis, ethyl methanesulfonate (EMS), an alkylating agent, offers an efficient and potent approach, creating single base pair changes or SNPs by acting primarily on guanine residues. These SNPs may either occur in coding regions, thus changing the encoded amino acids (non-synonymous), be silent (synonymous) or occur in non-coding regions, where they may induce changes in the promotor sequences or regulatory regions as well as intergenic regions without representing any measurable phenotype (Sikora et al., 2011). Non-synonymous SNPs can be used as markers for association studies to detect genetic

\footnotetext{
${ }^{1}$ http://www.kazusa.or.jp/jatropha/
}

variations related to phenotypic traits. They may also influence gene expression (up- or down regulation of gene transcription), and therefore represent a valuable tool for plant improvement (Ng and Henikoff, 2001).

Significant efforts toward large-scale characterization, screening, identification and discovery of SNP-mutants, e.g., DNA chips and microarrays and Targeting Induced Local Lesions In Genomes (TILLING) in combination with Next Generation Sequencing (NGS) have been used with success in crop plants (Till et al., 2007; Tsai et al., 2011; Maghuly et al., 2015, 2016, 2017). However, techniques that use quantitative polymerase chain reaction (PCR) only work for small fragments of DNA, array platforms are expensive and not flexible, and although sequencing candidate genes is the most accurate and straightforward alternative, it is relatively expensive (Sidhu et al., 2015). On the other hand, several genome-wide genotyping technologies have been developed and commercialized for SNP allele calling, e.g., Whole Genome Sequencing (WGS), RNA Sequencing (RNA Seq), exome capture, Restriction-site Associated DNA Sequencing (RADSeq), and Genotyping By Sequencing (GBS). RADSeq and GBS depend on Restriction Enzyme (RE) digestion. In contrast to WGS, they represent efficient and cost-effective methods for a large number of individuals to produce genetic information by applying efficient pooling strategies and reducing the representation of the genome (Beissinger et al., 2013). Further, RADSeq focusses on high target coverage of DNA fragment sequencing, while GBS involves sequencing DNA fragments at low target coverage and minimizing reads in repetitive sequences (Beissinger et al., 2013). The fact that GBS avoids random shearing and does not need size selection, nor specific library preparation, is completed in only two steps on plates followed by PCR amplification of the pooled library and is very simple compared to RADSeq, makes it a cost-effective alternative (Chen et al., 2013). In fact, low coverage genotyping methods produce large proportion of missing data (Wickland et al., 2017). The nGBS approach represents the genomic sequence between restriction sites by a single RE (Elshire et al., 2011), while double digested GBS (ddGBS), an alternative method to GBS, uses two enzymes, which can generate a suitable and uniform complexity reduction (Poland et al., 2012). Since the selection of optimal REs is critical for this method, some previous reports applied whole genome sequence data for in silico prediction of ddGBS to identify the size, number and genome position of digested fragments (Shirasawa et al., 2016).

However, besides different methods for identifying the effective mutation, it is of utmost necessity to use screening techniques for dissociation of chimeras from stable mutants. For somatic mutations, few methods exist for isolation of induced mutation or to produce plants without chimeric structure (Forster et al., 2012). Among them, in vitro culture techniques like protoplast, cell-, tissue- and organ- culture appear to be a fast, direct and robust method for isolating mutations (Suprasanna and Nakagawa, 2012). However, in vegetatively propagated plants, several cycles of regeneration are required to reduce the chimeras. If the optimal number of in vitro sub-culture was not achieved, the mutant would be chimeric, and the mutation of 
interest could be lost through subsequently multiplications (Shu et al., 2012).

In the current study, the development of a population of chemically induced mutants of J. curcas is described, which was screened for SNPs and InDels and subjected to functional analysis studies. To screen for rare mutations in genes of interest and to understand in silico gene function prediction on a whole-genome scale, two GBS based methods, namely normalized (n)GBS and double digest (dd)GBS were compared on 82 mutants and 14 wild-type accessions of J. curcas. Results show that nGBS-analyses of J. curcas mutants could be used as a reverse genetic method for genome-wide discovery of SNP loci even in crops with low genetic diversity.

\section{MATERIALS AND METHODS}

\section{Mutant Population}

A mutant population of 1,000 seeds from a selected J. curcas tree originated from Ethiopia was generated using three concentrations $(0.4,0.8$, and $1.6 \%)$ of the chemical mutagen ethyl methanesulphonate (EMS) for 3 different durations (0.5, 1.5 , and $3 \mathrm{~h}$ ) (Maghuly et al., 2017). The mutated population $\left(\mathrm{M}_{1} \mathrm{~V}_{1}\right)$ was transferred to appropriate tissue culture media and kept in a growth chamber with $12 \mathrm{~h}$ light at $28^{\circ} \mathrm{C}+-2^{\circ} \mathrm{C}$ (da Câmara Machado et al., 1997; Maghuly et al., 2017), at the PBU, BOKU University, Vienna, Austria. Control samples, not exposed to EMS treatment were grown in the same conditions. To reduce chimeras, subcultures were repeated every 3 weeks by the division of apical and lateral meristems. Following this process, the mutant lines from $\mathrm{M}_{1} \mathrm{~V}_{6}$ were subjected to genotypic analyses. Out of them, 96 samples containing 14 non-mutated wild-type J. curcas accessions and 82 mutated accessions were selected for the following studies (Supplementary Table S1).

\section{DNA Extraction}

$100 \mathrm{mg}$ of in vitro fresh young leaf tissue of each $\mathrm{M}_{1} \mathrm{~V}_{6}$ plants was used to extract total genomic DNA using DNeasy Plant Mini kit (Qiagen) following the supplier's instructions. The quality and concentration of DNA of each sample were determined using both gel electrophoresis and spectrophotometry.

\section{Normalized Genotyping by Sequencing (nGBS) Using MsII}

Genomic libraries were prepared following the protocol described by Elshire et al. (2011). Two hundred nano gram of each genomic DNA was digested with 1 Unit RE MslI (New England Biolabs, NEB) and $1 \times$ NEB buffer 4 for $1 \mathrm{~h}$ at $37^{\circ} \mathrm{C}$ in $30 \mu \mathrm{l}$ volumes. Incubation at $80^{\circ} \mathrm{C}$ for $20 \mathrm{~min}$ inactivated the REs.

The Encore Rapid Multiplex System was used to prepare Indexed Illumina libraries in a 96-well PCR plate by mixing $15 \mu \mathrm{l}$ of each digested DNA with $3 \mu \mathrm{l}$ of L2 Ligation adaptors, and $12 \mu \mathrm{l}$ master mix (4.6 $\mu$ l D1 water, $6 \mu$ l L1 Ligation Buffer, $1.5 \mu$ l L3 Ligation Enzyme). The reaction of ligation was completed at $25^{\circ} \mathrm{C}$ for 15 min. Next, $20 \mu$ l Final Repair Master Mix was added to each well, and the reaction was incubated at $72^{\circ} \mathrm{C}$ for $3 \mathrm{~min}$.
Fifty microliter of TE buffer (10 mM Tris/HCl, 50 mM EDTA, $\mathrm{pH} 8$ ) were added in tubes with reactions, which mixed with $80 \mu \mathrm{l}$ Agencourt XP beads (Beckman Coulter), incubated for $10 \mathrm{~min}$ at RT and placed the tubes on a magnet to collect the beads after $5 \mathrm{~min}$. The beads were washed two times with $200 \mu \mathrm{l} 80 \%$ Ethanol, after discarding the supernatant. Beads were air dried for $10 \mathrm{~min}$, and libraries were eluted in $20 \mu \mathrm{l}$ Tris buffer $(5 \mathrm{mM}$ Tris/ $\mathrm{HCl}, \mathrm{pH} 9)$. Ten microliter of each library were amplified in $20 \mu \mathrm{l}$ PCR reactions using MyTaq (Bioline) and standard Illumina TrueSeq amplification primers. The cycle number was limited to 10 cycles.

Five microliter of all 96 amplified libraries with different adaptors were pooled, and PCR primers, as well as small amplicons, were removed by 0.6 volume of beads using Agencourt AMPure XP PCR purification kit (Beckman Coulter). Further, the PCR enzyme was removed by an additional purification step on MinElute column (Qiagen). The pooled libraries were eluted up to $20 \mu \mathrm{l}$ by Tris Buffer $(5 \mathrm{mM}$ Tris/ $\mathrm{HCl}$, $\mathrm{pH}$ 9).

One microgram pooled GBS library was normalized using the Trimmer Kit (Evrogen) by mixing $12 \mu \mathrm{l}$ water and $4 \mu \mathrm{l}$ of $4 \times$ hybridization buffer. The prepared mix was denatured at $98^{\circ} \mathrm{C}$ for $3 \mathrm{~min}$ and renaturated at $68^{\circ} \mathrm{C}$ for $5 \mathrm{~h}$; after that $15 \mu \mathrm{l}$ of $2 \times$ DSN buffer was added and continued with incubation at $68^{\circ} \mathrm{C}$ for $10 \mathrm{~min}$. The reaction was treated with one Unit of DSN enzyme $(1 \mathrm{U} / \mu \mathrm{l})$ to degrade double-stranded DNA and incubated at $68^{\circ} \mathrm{C}$ for $30 \mathrm{~min}$. The reaction was terminated using $20 \mu \mathrm{l} \mathrm{DSN}$ Stop Solution, purified on a Qiagen MinElute column and eluted in $10 \mu \mathrm{l}$ Tris Buffer ( $5 \mathrm{mM}$ Tris/ $\mathrm{HCl} \mathrm{pH} \mathrm{9).}$

The Emulsion PCR (emPCR) was carried out for library re-amplification using buffers and enzymes from the emPCR Kit (Roche 454). The oil-surfactant mixture for creating the emulsion, columns and buffers for post-emPCR purification were provided by the Micellula DNA Emulsion \& Purification Kit (EURx). PCR water phase was produced by $5 \mu \mathrm{l}$ normalized DNA, $20 \mu \mathrm{l}$ of $5 \times$ Amp Mix, $40 \mu \mathrm{l}$ of emPCR Additive, $20 \mu \mathrm{l}$ of BiostabII PCR Enhancer (Sigma), $4 \mu \mathrm{l}$ of BSA $(10 \mathrm{mg} / \mathrm{ml}$, New England Biolabs, NEB), $4 \mu \mathrm{l}$ of TruSeq primer (50 pM/ $\mu \mathrm{l}$ each), $5 \mu \mathrm{l}$ of emPCR enzyme Mix and $0.2 \mu \mathrm{l}$ of Ppiase. An oil surfactant phase was created by $440 \mu \mathrm{l}$ of Emulsion Component $\mathrm{T}-1,40 \mu \mathrm{l}$ of Emulsion Component T-2 and $120 \mu \mathrm{l}$ of Emulsion Component T-3. One hundred microliter of PCR water phase and $600 \mu \mathrm{l}$ of oil surfactant phase were mixed in a $1.5 \mathrm{ml}$ reaction tube and vortexed for $5 \mathrm{~min}$ to generate a stable emulsion. Hundred microliter of the mix were used for PCR amplification. PCRcycling conditions consisted of $93^{\circ} \mathrm{C}$ for $1 \mathrm{~min}$ and continued by 18 cycles of $93^{\circ} \mathrm{C}$ for $30 \mathrm{~s}, 60^{\circ} \mathrm{C}$ for $30 \mathrm{~s}$ and $68^{\circ} \mathrm{C}$ for $2 \mathrm{~min}$. The breaking of the emulsion and subsequent DNA purification was done according to the Micellula DNA Emulsion \& Purification Kit manual (EURx). The nGBS library was finally size selected (200-500 bp) on a LMP-Agarose gel.

\section{Double Digest GBS (ddGBS) Library Construction Using Pst I and Msp I}

The ddGBs was performed as described by Poland et al. (2012). $200 \mathrm{ng}$ of genomic DNA were digested with 10 U HF-PstI (High 
Fidelity) and $8 \mathrm{U}$ MspI (New England Biolabs, NEB) in $20 \mu \mathrm{l}$ reaction volume of $1 \times \mathrm{NEB}$ buffer 4 for $2 \mathrm{~h}$ at $37^{\circ} \mathrm{C}$, followed by inactivation of the enzymes at $65^{\circ} \mathrm{C}$ for $20 \mathrm{~min}$.

The ligation reaction was carried out using $1 \times \mathrm{NEB}$ buffer 4, ATP, respective Adapter as described by Poland et al. (2012), containing a mix of common $\mathrm{Y}$ shaped adapter and sample specific barcoded adapter and T4 DNA Ligase. The ligation was carried out at $22^{\circ} \mathrm{C}$ for $2 \mathrm{~h}$ and enzyme was inactivated at $65^{\circ} \mathrm{C}$ for $20 \mathrm{~m}$.

Reactions were diluted with $50 \mu \mathrm{l}$ TE buffer $(10 \mathrm{mM}$ Tris/ $\mathrm{HCl}$, $50 \mathrm{mM}$ EDTA, pH 8.0) and mixed with $80 \mu \mathrm{l}$ Agencourt AMPure XP beads (Beckman Coulter), incubated for $10 \mathrm{~min}$ at RT and placed for $5 \mathrm{~min}$ on a magnet to collect the beads. After discarding the supernatant, the beads were washed two times using $200 \mu l$ of $80 \%$ Ethanol. Beads were air dried for $10 \mathrm{~min}$, and libraries were eluted in $10 \mu \mathrm{l}$ Tris Buffer ( $5 \mathrm{mM}$ Tris/ $\mathrm{HCl} \mathrm{pH}$ 9).

Three microliter of each library were separately amplified in $20 \mu 1$ PCR reactions using Herculase II (Agilent Technologies). Cycle number was limited to 14 Cycles.

All 96 amplified samples were pooled, mixed, and purified using MinElute PCR Purification Kit (Qiagen). Concentration was measured on Qubit, and the quality checked on an Agilent High Sensitivity DNA Chip. The GBS library was finally size selected (200-500 bp) on a LMP-Agarose gel.

\section{Data Analysis, Read Preprocessing, GBS Alignment and SNP Discovery}

The libraries were sequenced using Illumina HiSeq 2000 and paired-end sequence reads from FASTQ files were demultiplexed with Illumina's CASAVA data analysis software and software developed by LGC Genomics. All the raw sequencing reads were deposited in the Short Read Archive (SRA) database is retrievable under the accession number SRP136238 in the SRA database of NCBI.

Reads were discarded using the following criteria (a) reads containing unspecified nucleotides (Ns), (b) reads with a final length of less than 20 bases and (c) reads where the $5^{\prime}$ end did not match with the RE site. Reads were quality trimmed at $3^{\prime}$ end and reads with a final length less than 64 bases were discarded. For all FASTQ files, FastQC reports were created. BWA version $0.7 .5 \mathrm{a}^{2}$ was used to map (aln/sampe) trimmed reads against the reference genome of $J$. curcas JAT r4.5. ${ }^{3}$ Variant discovery and genotyping of samples was performed with Freebayes v0.9.9 $9^{4}$ using specific parameters "-min-base-quality 10 -min-supporting-allele-qsum 10 read-mismatch-limit 3 -min-coverage 5 -min-alternate-count 4 -report-genotype-likelihood-max -exclude-unobservedgenotypes -genotype-qualities -no-mnps -no-complex ploidy 2."

Genotype calls were filtered with the following GBSspecific filter rule set: (a) including only variants for which both alleles have been called, (b) genotypes must have been observed in at least $66 \%$ of the samples, (c) minimum

\footnotetext{
${ }^{2} \mathrm{http}: / /$ bio-bwa.sourceforge.net/

${ }^{3} \mathrm{ftp}: / / \mathrm{ftp}$.kazusa.or.jp/pub/jatropha/

${ }^{4}$ https://github.com/ekg/freebayes
}

total depth across all samples must exceed 20 reads, (d) minimum allele depth across all samples must exceed 10 reads, (e) sample genotype data where total read count was below 8 were removed, (f) heterozygous calls were reverted to homozygote calls when the allele balance was outside 20$80 \%$.

Variants with only heterozygous calls were excluded. Annotation of variant effect on genes and transcripts was performed using SnpEff version $3.2^{5}$ and the Blast2GO annotation tool (Conesa et al., 2005). The predicted genes and transcripts according to the genome annotation were used to predict downstream functional effects of the SNPs.

\section{Reference Sequences}

Sequence and annotation of $J$. curcas JAT r $4.5^{3}$ were used for alignment, and a manually improved GTF file (derived from the provided GFF file) was used with SnpEff to annotate putative functional effects of the variants.

\section{RESULTS}

To screen for novel mutations in genes of interest and to understand in silico gene function prediction on a whole-genome scale, nGBS and ddGBs based on Illumina sequencing (by LGC Genomics) were used on 82 mutants and 14 wild-type accessions of $J$. curcas.

To make GBS libraries of $J$. curcas, containing a small size genome $(1 \mathrm{C}=416 \mathrm{Mb})$, and to maximize the desired size distribution (200-500 bp) of restriction fragments for sequencing library, different REs were selected. The GBS libraries were prepared and sequenced from the same set of DNAs using MslI for nGBS and PstI/MspI for ddGBS with 10, 6 and 4-base recognition sites, respectively.

\section{Structure and Functional Annotation of nGBS-Based Variants}

Genomic DNA digested with MslI and fragments in the desired size range was recovered in libraries from 96 different individuals tagged with 96 barcodes. Sequencing of 96 samples on Illumina HiSeq 2000 platforms produced 263,340,722 of 100 -bp paired-end raw reads. Figure 1 describes all steps carried out for reading processing. After the low-quality reads were trimmed and filtered, a total of 159,380,823 high-quality reads remained. The average number of reads per individuals was 1,656,035 million, ranging between 49,564 (MslI-D06) and 2,459,394 million reads (MslI-E09). The number of reads among EMS mutant plants ranged from 49,564 (MsII-D06) to 2,459,394 (sample MsII-E09), whereas the number of reads for the wild-type J. curcas ranged from 1,195,496 (MsII$\mathrm{H} 03$ ) to 2,418,812 (MsII-G03) (Supplementary Table S2). The provided sequencing reads were mapped to the publicly available reference sequence ${ }^{3}$. Further, additional stringent filters were applied for variant analysis, which removed variations with a

${ }^{5} \mathrm{http}: / /$ snpeff.sourceforge.net/ 


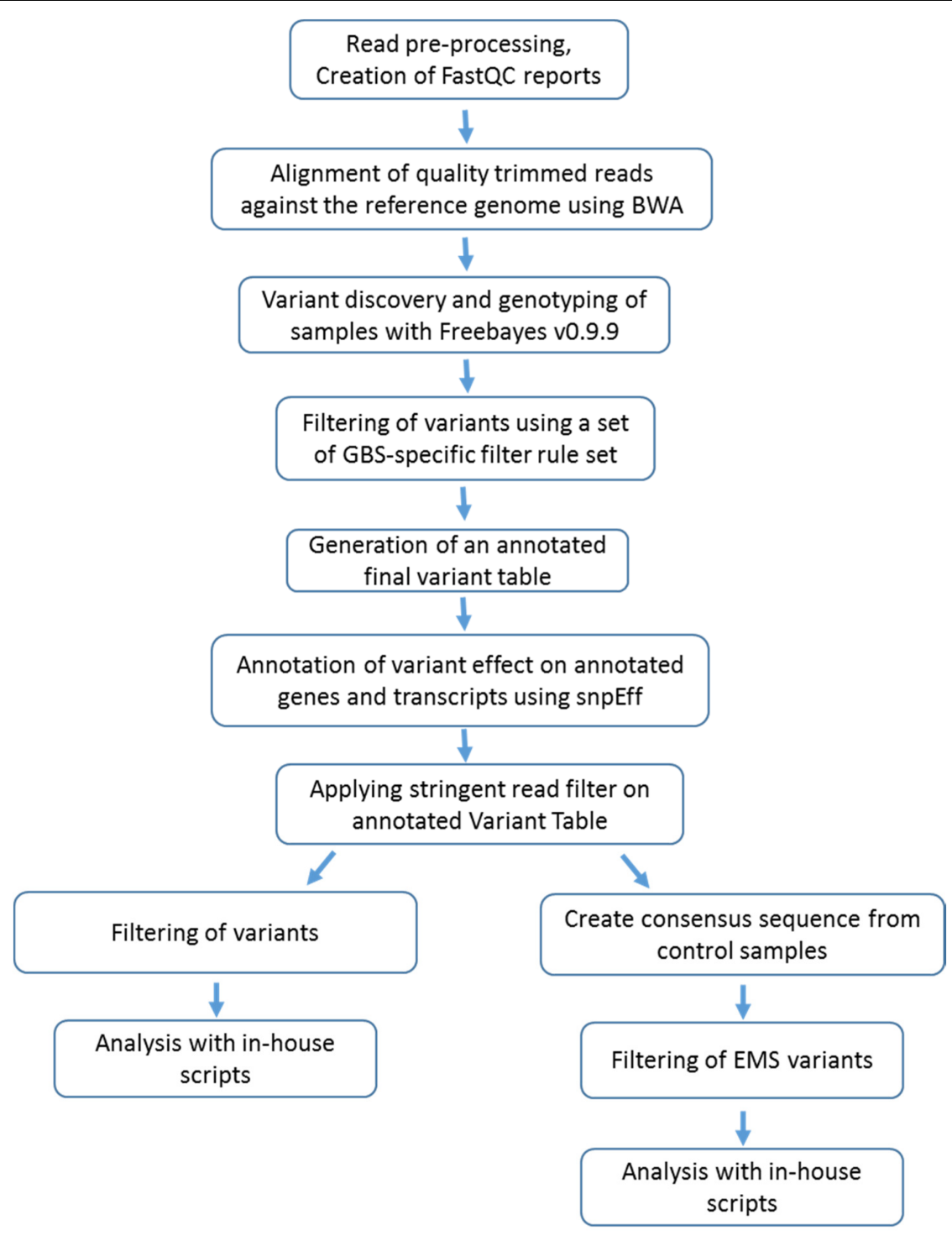

FIGURE 1 | Bioinformatics workflow that was used in this study.

total read count less than 20 and a minor allele count less than 10 .

Data analyses among $96 \mathrm{~J}$. curcas samples identified 79,688 SNPs (62,418 heterozygous), 1,567 InDels and 155,831 missing alleles. The InDels ranged from 2 to $5 \mathrm{bp}$, with the majority of mononucleotide InDels (Supplementary Table S3).

The total number of heterozygous and homozygous SNPs for alternative alleles per variant ranged from 184 (MsII-G11) to 1,059 (MsII-E09) and from 29 (MsII-G11) to 310 (MsII-E09), respectively. Due to the small number of reads, the sample MSII-D06 was removed (Supplementary Tables S2, S4).

Further, 1,366 variants were found in coding regions, 421 in introns, 1,541 in intergenic regions, and 1,874/1,723 were located in up- and downstream regions, respectively. The functional effect of SNPs and InDels in genic regions on 945 annotated genes (predicted protein sequences) of the reference genome were classified by the SnpEff program ${ }^{6}$. Among them, 771 were nonsynonymous mutations, 507 synonymous, whereas only a small fraction of mutations (18) induced frame-shifts. Of these, we could identify a few causing a loss of start and stop (3 and 7 respectively), and a somewhat higher occurrence in stop gained (60). Further, 8 effects were found in splice site donor and 9 in splice site acceptor (Table 1). The details regarding functional annotation of the relevant gene derived non-synonymous SNPs are shown in Table $\mathbf{1 .}$

Depending on the type of nucleotide substitutions, SNPs were classified into transitions (Ts) and transversions (Tv), using VCFtools (Table 2). Most of the SNPs were Ts $(53,621)$, with the C/T (51\%) and G/A (49\%) transitions accounting for $67.8 \%$ of the SNPs. The other four SNP types were Tv $(25,406)$, which includes

${ }^{6} \mathrm{http}: / /$ snpeff.sourceforge.net 
TABLE 1 | Genomic features (classification) of SNP and InDel distributions identified by nGBS.

\begin{tabular}{lcc}
\hline Effect & $\begin{array}{c}\text { SNP and InDel } \\
\text { occurrences }\end{array}$ & $\begin{array}{c}\text { Number of distinct } \\
\text { genes }\end{array}$ \\
\hline Synonymous & 507 & 366 \\
Non-synonymous & 771 & 501 \\
Stop gained & 60 & 52 \\
Stop lost & 7 & 7 \\
Start lost & 3 & 2 \\
Frame-shift & 18 & 17 \\
Intron & 421 & 0 \\
Intergenic & 1,541 & 0 \\
Upstream & 1,874 & 1,268 \\
Downstream & 1,723 & 1,143 \\
Splice site acceptor & 9 & 3 \\
Splice site donor & 8 & 5
\end{tabular}

TABLE 2 | Number of transitions and transversions identified by nGBS.

\begin{tabular}{lc}
\hline Substitution & Number of SNPs \\
\hline Transitions & 53,621 \\
C/T & 27,438 \\
A/G & 26,183 \\
Transversions & 25,406 \\
C/G & 6,366 \\
A/T & 6,321 \\
A/C & 6,660 \\
G/T & 6,059 \\
TS/Tv ratio & 2.1 \\
\hline
\end{tabular}

C/G (25\%), G/T (24\%), C/A (26\%) and A/T (25\%) transversions accounting for $32.2 \%$ of all SNPs. The Ts/Tv ratio in this study was 2.1 (Figure 2_nGBS and Table 2).

In category Biological Process (BP), functional annotation in genic regions of variable alleles of 235 genes with severe effects were classified in 19 different GO pathways, of which a significant portion was annotated as genes performing DNA integration (335 SNPs and 9 InDels) and protein phosphorylation (13 SNPs). Of the 397 high impact variations, 388 SNPs and 9 InDels were identified, which contain 356 SNPs with non-synonymous effect, 29 stop-gained, 9 frame-shift and 3 stop-lost.

By category, Molecular Function (MF), 41 GO pathways containing 490 SNPs and 13 InDels related to 303 genes were annotated, the highest number of variable alleles belong to the nucleic acid binding and zinc ion binding (386 nonsynonymous, 30 stop-gained, 2 stop-lost, and 13 InDels). Out of 503 mutations (SNPs and InDels), 452 were annotated to have a non-synonymous, 34 a stop-gained, 3 a stop-lost and 13 a frame-shift effect. The least GO terms (8) were found under the category Cellular Component (CC) with 10 genes containing 17 SNPs. Out of them, 16 mutation changes showed non-synonymous, and one-stop gained effect (Supplementary Figure S1 and Supplementary Table S5).

To reconstruct the metabolic pathways, the variable alleles in genic regions were mapped to KEGG pathways

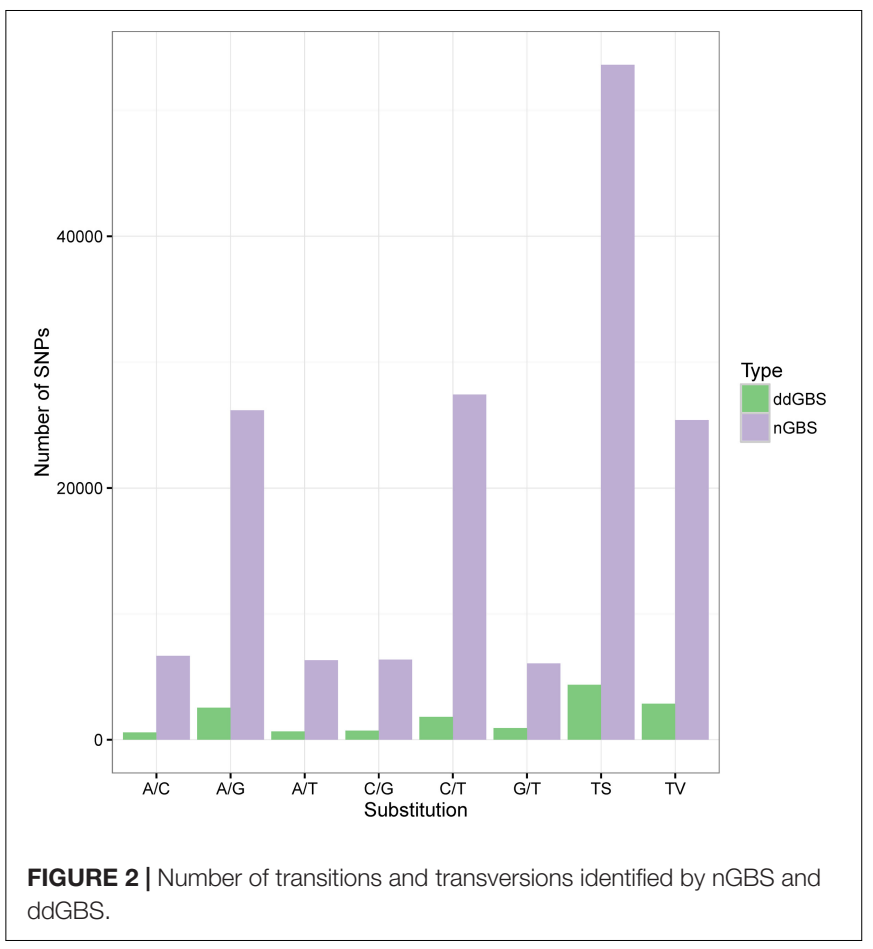

(Sidhu et al., 2015). In total 16 SNPs (14 with non-synonymous and 2 with stop-gained effects) were located on six KEGG pathways (Supplementary Table S6). The most highly represented pathways were ether-lipid metabolism and glycerophospholipid metabolism each with 5 SNPs affecting the same gene (Jcr4S00177.210), followed by starch and sucrose metabolism with 3 SNPs on three different genes. These results showed that identified regions near variable changes could modify the oil and polysaccharide contents, which can be evaluated by various metabolic analyses.

\section{Detection of EMS-Induced Changes by nGBS}

To identify SNPs that were introduced by EMS we compared the non-mutated accessions to the mutated samples. Due to the heterogeneous genetic background of the 14 non-mutated accessions, we implemented a novel approach to build a consensus sequence. The consensus sequence based on 14 wild type $J$. curcas accessions was predicted using the variants that all 14 accessions had in common leading to a reduction of genomic positions to 365 . Here 340 consensus sequences are homozygous to the reference and 25 are homozygous to the alternate allele. The final consensus sequence was used for direct comparison of mutant and non-mutant accessions.

Using this consensus sequence, 365 distinct genomic positions could be used for comparison totaling 29,930 positions amongst all 82 mutated samples.

Genotypes varying from the consensus were defined as an EMS-induced mutation. In total 1,014 EMS induced mutations were identified whereas 27,810 positions had the same alleles/SNPs as the consensus sequence. The remaining 1,106 
positions were removed because of low read count or missing data (Supplementary Tables S7, S8). Overall, SNP changes were distributed in 427 annotated genes. In total, 207 EMS-induced mutations were identified in coding regions and 267 SNPs in intergenic regions followed by 74 in upstream, 29 in downstream and 45 in intron regions.

For individual plants, the number of positions equal to the consensus sequence ranged from 214 in MSII-G11 to 359 in MSII-C09. Of the EMS mutational changes, the smallest number of SNPs was found in sample MSII-B03 with 5 sequence changes, followed by MSII-C08 with 6 changes. The highest number of EMS-induced SNPs was observed in sample MSII-C09 with 38 changes, followed by MSII-C12 with 28 changes (Supplementary Table S8). Interestingly, the samples with highest and lowest EMS mutations are related to the treatment group with $0.8 \%$ EMS and $1.5 \mathrm{~h}$.

Functional annotation of SNPs in coding regions with high to moderate effects (non-synonymous, stop lost, stop gained, start lost, frame-shift) revealed 40 SNPs in 30 different genes with 38 non-synonymous and 2 stop-gained effects involved in two separate pathways (DNA integration and intracellular protein transport) of BP category (Supplementary Figure S2 and Supplementary Table S9).

In the category MF, 53 SNPs were located in 42 different genes with 2 stop-gain and 40 non-synonymous effects involved in 8 different pathways. The largest proportion of SNPs are effecting the nucleic acid binding and zinc ion binding pathways. Further, the only SNP in CC category effecting the "clathrin adaptor complex; Golgi apparatus" pathway was identified in sample MSII-E01 (Supplementary Figure S2 and Supplementary Table S9).

\section{Structure and Functional Annotation of ddGBS-Based Variants}

Genomic DNA of 96 different individuals was double-digested with REs PstI and MspI and fragments in a size range of 200-500 bp and sequenced on Illumina HiSeq 2000 platform. Sequencing of 96 samples yielded 135,531,226 raw reads. After trimming and filtering the low-quality reads, 118,258,489 highquality trimmed reads were obtained. The average number of high quality reads per individuals was 1,231,859 reads ranging between 171,348 (PstI-MspI-A04) and 1,798,892 (PstI-MspI-G06) reads. The number of reads among EMS mutant plants ranged from 309,172 (PstI-MspI-A10) to 1,798,892 (PstI-MspI-G06), whereas the number of reads for the non-mutated plants ranged from 171,348 (PstI-MspI-A04) to 1,603,586 (PstI-MspI-G04) (Supplementary Table S10).

The produced sequence reads were mapped to reference sequences generated from the publicly available data ${ }^{7}$ (Supplementary Table S11). In the 96 J. curcas samples, 5,525 heterozygous SNPs and 623 InDels were identified. Also, 3,911 were homozygous to the reference, 1,794 were homozygous to the alternate alleles, and 8,787 were missing. The InDels ranged from 2 to $4 \mathrm{bp}$, with the majority of mononucleotide InDels. The identified SNPs and InDels in genic regions were distributed

${ }^{7} \mathrm{ftp} / / / \mathrm{ftp}$.kazusa.or.jp/pub/jatropha/ across 68 genes (Table 3). The total number of heterozygous, homozygous for alternative alleles SNPs and InDels per genomic positions ranged from 15 (PstI-MspI A10) to 101 (PstI-MspI E09) and 4 (PstI-MspI A04) to 26 (PstI-MspI D04, Pst I-MspI G07), respectively. These EMS mutated samples originated from different EMS concentrations, i.e., $0.8 \% 3 \mathrm{~h}$ (PstI-MspI A10, PstI-MspI E09, PstI-MspI G07) and 1.6\% $3 \mathrm{~h}$ (PstI-MspI D04) (Supplementary Table S12).

Further, 60 SNPs and InDels were found in introns, 58 in intergenic and 86 in coding regions (Table 3 ). Of the changes affecting the coding regions, 30 were synonymous and 54 non-synonymous, which were identified in 27 and 40 genes, respectively (Table 3 ). They had very low occurrences in splice site acceptors and stopped lost (a single mutation for each), and higher occurrences in down- and upstream regions (104, 101, respectively).

Most of the identified SNPs were transitions $(4,360)$, with the C/T (42\%) and G/A (58\%) transitions accounting for $60.4 \%$ of the SNPs. On the other hand, 2,859 transversions were identified, which include C/G (25\%), G/T (32\%), C/A (20\%) and A/T (23\%) accounting for $39.6 \%$ of all SNPs (Figure 2 _ddGBS and Table 4). The Ts/Tv ratio in these samples was 1.5 .

Functional annotation categorized 22 non-synonymous SNPs located on 16 genes in $6 \mathrm{GO}$ pathways of BP. In the category MF, 23 GO pathways with 42 SNPs on 31 genes were identified, containing 41 non-synonymous and one-stop lost. The lowest number of SNPs (6) were found in category CC, with 4 GO pathways and five genes.

TABLE 3 | Genomic features (classification) of SNP and InDel distributions identified by ddGBS.

\begin{tabular}{lcc}
\hline Effect & $\begin{array}{c}\text { SNP and InDel } \\
\text { occurrences }\end{array}$ & $\begin{array}{c}\text { Number of distinct } \\
\text { genes }\end{array}$ \\
\hline Synonymous & 30 & 27 \\
Non-synonymous & 54 & 40 \\
Stop lost & 1 & 1 \\
Codon insertion & 1 & - \\
Intron & 60 & - \\
Intergenic & 58 & - \\
Upstream & 101 & 72 \\
Downstream & 104 & 78 \\
Splice site acceptor & 1 & 1 \\
\hline
\end{tabular}

TABLE 4 | Number of transitions and transversions identified by ddGBS.

\begin{tabular}{lc}
\hline Substitution & Number of SNPs \\
\hline Transitions & 4,360 \\
$\mathrm{C} / \mathrm{T}$ & 1,816 \\
$\mathrm{~A} / \mathrm{G}$ & 2,544 \\
Transversions & 2,859 \\
$\mathrm{C} / \mathrm{G}$ & 715 \\
$\mathrm{~A} / \mathrm{T}$ & 651 \\
$\mathrm{~A} / \mathrm{C}$ & 574 \\
$\mathrm{G} / \mathrm{T}$ & 919 \\
Ts/Tv ratio & 1,5
\end{tabular}


In the category BP, the highest number of SNPs (10) were involved in oxidation-reduction process, followed by 7 SNPs related to protein phosphorylation, while in the MF category 9 SNPs in five genes were also involved in oxidoreductase activity, performing with integration or reduction of molecular oxygen, iron and heme binding, followed by 4 SNPs in 3 genes associated with transferase activity. The type of variation, the location on genes and samples containing are described in the Supplementary Figure $\mathbf{3}$ and Supplementary Table S13.

One non-synonymous SNP, located on gene Jcr4S03126.20 and identified by KEGG analysis, was belonging to two pathways (sesquiterpenoid and triterpenoid biosynthesis, steroid biosynthesis) (Supplementary Table S14). Steroid hormones, which are important for plant growth and fruit development, are produced during steroid biosynthesis pathway via MAV (Mevalonate) pathway. On the other hand, triterpenoids and sesquiterpenoids, which have a significant role in biotic and abiotic stress response, are also generated via MVA pathway. These data could be supported by previous studies that identified region harboring variable changes involved in stress response (Zmienko et al., 2014; Ariani et al., 2016).

Efforts to identify EMS-induced changes by ddGBS, however, yielded no results.

\section{DISCUSSION}

Natural as well as induced mutations, which are responsible for genetic variation, are the raw material for plant breeding (Jiao et al., 2016). Recent studies showed the genetic diversity within J. curcas accessions to be narrow, resulting from genetic history, population bottlenecks, lack of evolutionary change or a high level of human-driven selection, which may have decreased the genetic diversity in J. curcas and increased its homogeneity (Doebley et al., 2006; Achten et al., 2010; Maghuly et al., 2015). Also, J. curcas is vegetatively propagated, which further reduces the genetic variation (Ambrosi et al., 2010). Further, the mating system plays a key role in homogeneity and low genetic diversity in J. curcas, being a mixed mating system through self- and cross-pollination, with high rates of full-sib seeds (Bressan et al., 2013; Negussie et al., 2014). On the other hand, because Jatropha species are often restricted to Isolated sites, where they adapted to a particular climate or soil types, the frequency of interbreeding is low, which may have additionally reduced the genetic diversity (Alves et al., 2013). In such cases inducing genetic diversity by generating a mutant population from a uniform genetic background like Jatropha is a robust strategy to create novel variations for crop improvement in plant breeding programs and to connect genotypic variation to phenotypic diversity (Jiao et al., 2016).

For Jatropha, so far, only a few studies have applied physical and chemical mutagenesis for its improvement, e.g., different doses of $\gamma$-rays $(5-50 \mathrm{Kr})$ were reported to change various morphological traits in Jatropha. Unfortunately, these studies were only verified in $\mathrm{M}_{1}$ populations, without any reverse genetic analyses (Dhakshanamoorthy et al., 2011; Dhillon et al., 2014). Also, different concentrations of EMS (1-4\%) were used in J. curcas (Dhakshanamoorthy et al., 2011), but only random amplified polymorphic DNA (RAPD) markers were used to identify polymorphisms.

The effect of the artificial mutation can be detected by different approaches, like the observation of phenotypic variation (forward genetics) (Mohd-Yusoff et al., 2015), however, in some case, phenotypic variations are not visible, since they may affect physiological and biological processes. For examples, in EMS-induced Arabidopsis mutants response to high temperature was affected through decreasing in $\mathrm{ABA}$, and salicylic acid synthesis (Zhu et al., 2012), sensitivity to ABA was reduced in chemically mutated L. japonica (Biswas et al., 2009), in soybean TILLING an increasing in the oleic acid content was confirmed by identification of novel mutations in the FAD1, 2, and 3 genes (Dierking and Bilyeu, 2009) and in Sorghum the lignin content was decreased by mutation of the gene encoding caffeic acid $O$-methyltransferase (Xin et al., 2008).

In this case, the application of a cost-effective and highthroughput and efficient screening mutation (reverse genetics) method allowing to predict gene functions and to analyze the phenotypic consequences is advantageous. In fact, to identify novel traits in a large mutant population, and especially in plants without a complete reference genome, like Jatropha, a high throughput, cost-effective genetic screening technology is needed. Traditionally, TILLING is a popular method to identify causative SNPs in mutated population; however, it requires significant time and effort to design specific primers for selected genes (Sidhu et al., 2015). Next-generation sequencing experiments based genotyping is routinely used to find rare and novel variation and to detect mutations in various plant species (Sidhu et al., 2015). Among different reverse genetic approaches, GBS showed to be an efficient method to identify a mutation without prior knowledge of the target genes. It also proved to be a suitable method to determine SNPs and InDels, functional genes and the allele within a reasonably short period of time (Ariani et al., 2016). Therefore, for the first time, in the current study, the application of sequencing-based genotyping approaches was tested for the evaluation of vegetatively propagated J. curcas mutated by EMS.

During the experimental and data analyses process, we found that although the amounts of DNA per sample were equal, the number of generated reads for each sample and for each site was different, which also previously reported by (Elshire et al., 2011; Mascher et al., 2013; Liu et al., 2014; Sidhu et al., 2015). This could result in missing data and therefore has a significant effect on data analyses (Jiang et al., 2016). To overcome this limitation, it was suggested (Harloff et al., 2012) to add additional filter, use paired-end reads, and/or using alternative method to increase the site coverage and to generate more consistent results by introducing two enzymes like ddGBS (Peterson et al., 2012; Poland et al., 2012). In this case, selection and evaluation of the effect of the REs is critical, especially for plants with a high degree of DNA methylation like 
J. curcas (Yi et al., 2010). Interestingly, obtained result in the current study showed that the selected enzymes for ddGBS were not consistent with in silico simulation, since ddGBS analyses obtained lower numbers of fragment and SNPs compared two nGBS (Supplementary Table S15), resulting from the high frequency of DNA methylation. The reasons underlying the low number of fragments or missing data in ddGBS could be the following: (a) restriction sites are missing in samples because of biological cases or mutation (natural or induced mutation); (b) the DNA was not digested due to DNA methylation; (c) PCR was not as efficient for the specific fragment, and (d) low coverage of particular position, because of sequencing setup. However, the small number of reads and SNPs in the current study could be explained by the occurrence of high amounts of $\mathrm{CpNpG}$ in the Jatropha genome. In a survey based on methylation-sensitive fluorescence, (Yi et al., 2010) reported that more than half of the CCGG site found in J. curcas were methylated. In fact, most of the genetic variation reported for this species was shown to be mostly epigenetic (Carels, 2013). In plant systems, the most abundant context of methylation occurs within a C-G dinucleotide $(\mathrm{CpG})$, usually symmetrically on both DNA strands. Since plants contain high amounts of CpG, PstI, which is CpNpG sensitive, will generally be applied with other frequently cutting enzymes (4 cutters, e.g., $M s p$ I) for GBS analyses. Therefore, for the selection of a suitable enzyme combination for ddGBS, it is helpful to carry out whole-genome bisulfite sequencing or to perform a small-scale pilot experiment with several combinations of restriction enzyme (Shirasawa et al., 2016).

On the other hand, the common problem of reduced representation sequencing methods like GBS are high error rates in distinguishing heterozygous and homozygous individuals, which can conceal the identification of true variation (Nielsen et al., 2011; Sidhu et al., 2015; Jiang et al., 2016). To identify variation induced by EMS, we added an additional set of filter, by using the variants that all wild accessions, containing similar genetic background, had in common.

We also found that the mutation spectrum encountered in the current study was prevalently $\mathrm{G} / \mathrm{C}$ to $\mathrm{A} / \mathrm{T}$ transitions (60\% of base changes), which is in agreement with research carried out on barley (70\%) (Caldwell et al., 2004), rice (70\%) (Till et al., 2007), Lotus japanicus (65\%) (MohdYusoff et al., 2015) and tomato (60\%) (Minoia et al., 2010). Only in Arabidopsis (99\%) and banana (100\%) a higher frequency of $\mathrm{G} / \mathrm{C}$ to $\mathrm{A} / \mathrm{T}$ base changes were identified. Further, besides $\mathrm{G} / \mathrm{C}$ to $\mathrm{A} / \mathrm{T}$ transitions also transversions were found in EMS mutated Jatropha, which represent one-third of the total substitutions. It was previously suggested, that they should not be removed from a mutation study since they could be a causative mutation for a trait of interest (Mohd-Yusoff et al., 2015). As described before, causative mutations in coding regions can have either a synonymous or non-synonymous effect, the later disrupting protein-coding sequences (Sikora et al., 2011). The identification of nonsynonymous effects, as selected for functional annotation analyses, associated with phenotypic variation is of crucial importance.
Regardless of the method used for SNP detection, it is necessary to dissociate genotypic heterogeneity or chimeras, which occurs at the time of mutagenesis, to identify desirable genetically homogenous mutants. Chimerism normally is eliminated through sexual propagation by self-pollination of the mutated population $\left(\mathrm{M}_{1}\right)$ to produce the $\mathrm{M}_{2}$ or higher populations (Comai and Henikoff, 2006). In the current study, the reduction of chimeras was achieved through vegetative propagation of apical as well as lateral meristems. Since previous studies on the mutant population of banana suggested that most chimera should be dissolved when reaching $\mathrm{M}_{1} \mathrm{~V}_{3}$ and $\mathrm{M}_{1} \mathrm{~V}_{4}$ populations (Jankowicz-Cieslak et al., 2012), it was considered that $M_{1} V_{6}$ sibling should be free of chimerism.

\section{CONCLUSION}

In conclusion, a total of $96 \mathrm{M}_{1} \mathrm{~V}_{6}$ plants (wild-type and induced mutation populations) were selected, and the frequency and the effect of EMS treatments were compared using both nGBS and ddGBS analyses. As reverse genetic approaches, they represent an efficient method for discovery of the induced mutation in a large population with a large number of genes associated with phenotypic variation. Obtained data showed that allelic variations and in silico analysis of gene functions could be rapidly identified in mutant populations using nGBS and ddGBS. The results also confirmed the existence of high genome methylation in J. curcas, which could be responsible for its genome plasticity and ability to survive in different climate conditions. EMSinduced mutants exhibited a wide range of phenotypic variation, especially in leaf and stem architecture, which depends on the concentration of the applied mutagen (Maghuly et al., 2017).

A significant result of this study was to understand that the handling of GBS data is more difficult and more challenging than the traditional TILLING strategy in mutated plants, which is routinely used for reverse genetic analyses. Also, since it is difficult to get full coverage of potential protein-coding regions among all samples by these methods, it was also difficult to perform a study of average mutation density. Further, since the Jatropha genome sequence is incomplete and therefore contains missing genes, alignment and variant analysis of target sequence reads is challenging to perform and interpret. Thus, providing a complete Jatropha reference genome sequence with high quality should be a priority for any breeding program. However, increasing accessibility of high-throughput technology with decreasing cost of sequencing. Although in our data the ddGBS method showed low-density SNP markers and low genotyping accuracy, using suitable methylation sensitive or insensitive restriction enzymes will give the opportunity to use GBS with higher site coverage and lower genotyping error. At last, continuously vegetatively propagated tissue is a fast, efficient and accurate method to dissolve chimeras, especially for long-lived plants like J. curcas. Further, if the gene of interest contains a causative mutation, this should be validated. 


\section{AUTHOR CONTRIBUTIONS}

FM and ML designed the whole study, carried out the all experiments, participated in data analyses, and wrote the final manuscript. SP and JK participated in data analyses and manuscript editing. All authors read and approved the final manuscript.

\section{FUNDING}

This research was financially supported by the Austrian Science Fund (FWF, P 23836), FFG and Bioplant R\&D.

\section{SUPPLEMENTARY MATERIAL}

The Supplementary Material for this article can be found online at: https://www.frontiersin.org/articles/10.3389/fpls.2018.00524/ full\#supplementary-material

FIGURE S1 | Functional annotation of nGBS variants in genic regions in three GO categories (BP, MF, CC).

FIGURE S2 | Functional annotation of EMS-induced SNPs identified by nGBS in genic regions in three $\mathrm{GO}$ categories (BP, MF, CC).

FIGURE S3 | Functional annotation of ddGBS variants in genic regions in three $\mathrm{GO}$ categories (BP, MF, CC).

TABLE S1 | List of 96 samples containing 14 non-mutated wild-type accessions, 82 mutated accessions, and their treatments.

\section{REFERENCES}

Achten, W. M., Nielsen, L. R., Aerts, R., Lengkeek, A. G., Kjær, E. D., Trabucco, A., et al. (2010). Towards domestication of Jatropha curcas. Biofuels 1, 91-107. doi: 10.1016/j.tplants.2016.08.008

Alves, A. A., Bhering, L. L., Rosado, T. B., Laviola, B. G., Formighieri, E. F., and Cruz, C. D. (2013). Joint analysis of phenotypic and molecular diversity provides new insights on the genetic variability of the Brazilian physic nut germplasm bank. Genet. Mol. Biol. 36, 371-381. doi: 10.1590/S141547572013005000033

Ambrosi, D. G., Galla, G., Purelli, M., Barbi, T., Fabbri, A., Lucretti, S., et al. (2010). DNA markers and FCSS analyses shed light on the genetic diversity and reproductive strategy of Jatropha curcas L. Diversity 2, 810-836. doi: 10.3390/ d2050810

Ariani, A., Berny, J. C., and Gepts, P. (2016). Genome-wide identification of SNPs and copy number variation in common bean (Phaseolus vulgaris L.) using genotyping-by-sequencing (GBS). Mol. Breed. 36:87. doi: 10.1007/s11032-0160512-9

Bado, S., Forster, B. P., Nielen, S., Ali, A. M., Lagoda, P. J. L., Till, B. J., et al. (2015). "Plant mutation breeding: current progress and future assessment," in Plant Breeding Reviews, Vol. 39, ed. J. Janick (Hoboken, NJ: John Wiley \& Sons, Inc.).

Beissinger, T. M., Hirsch, C. N., Sekhon, R. S., Foerster, J. M., Johnson, J. M., Muttoni, G., et al. (2013). Marker density and read depth for genotyping populations using genotyping-by-sequencing. Genetics 193, 1073-1081. doi: 10.1534/genetics.112.147710

Biswas, B., Chan, P. K., and Gresshoff, P. M. (2009). A novel ABA insensitive mutant of Lotus japonicus with a wilty phenotype displays unaltered nodulation regulation. Mol. Plant 2, 487-499. doi: 10.1093/mp/ ssp009

Bressan, E., Sebbenn, A., Ferreira, R., Lee, T., and Figueira, A. (2013). Jatropha curcas L. (Euphorbiaceae) exhibits a mixed mating system, high correlated
TABLE S2 | Distribution of $\mathrm{nGBS}$ reads in each sample and their proportion mapped to the reference.

TABLE S3 | Annotation of nGBS variants on annotated genes and transcripts using SnpEff.

TABLE S4 | Distribution of SNPs and InDels identified by nGBS in each sample.

TABLE S5 | Functional annotation of nGBS variants in genic regions in three GO categories (BP, MF, CC).

TABLE S6 | The nGBS variable alleles in genic regions assigned to KEGG pathways.

TABLE S7 | Annotation of EMS-induced changes identified by the nGBS-based variant on annotated genes and transcripts using SnpEff.

TABLE S8 | Distribution of EMS-induced SNPs identified by nGBS in each sample.

TABLE S9 | Functional annotation of EMS-induced SNPs identified by nGBS in genic regions in three $\mathrm{GO}$ categories.

TABLE S10 | Distribution of ddGBS reads in each sample and their proportion mapped to the reference.

TABLE S11 | Annotation of ddGBS variants on annotated genes and transcripts using SnpEff.

TABLE S12 | Distribution of SNPs and InDels identified by ddGBS in each sample.

TABLE S13 | Functional annotation of ddGBS variants in genic regions in three GO categories (BP, FM, CC).

TABLE S14 | The ddGBS variable alleles in genic regions assigned to KEGG pathways.

TABLE S15 | Distribution of average number of reads and heterozygous SNPs for nGBS, ddGBS and each treatments.

mating and apomixis. Tree Genet. Genomes. 9, 1089-1097. doi: 10.1007/s11295013-0623-y

Caldwell, D. G., McCallum, N., Shaw, P., Muehlbauer, G. J., Marshall, D. F., and Waugh, R. (2004). A structured mutant population for forward and reverse genetics in Barley (Hordeum vulgare L.). Plant J. 40, 143-150. doi: 10.1111/j. 1365-313X.2004.02190.x

Carels, N. (2013). "Towards the domestication of Jatropha: the integration of sciences," in Jatropha, Challenges for a New Energy Crop, eds B. Bahadur, M. Sujatha, and N. Carels (New York, NY: Springer), 263-299. doi: 10.1007/978-14614-4915-7_14

Carvalho, C. R., Clarindo, W. R., Prača, M. M., Araújo, F. S., and Carels, N. (2008). Genome size, base composition and karyotype of Jatropha curcas L., an important biofuel plant. Plant Sci. 174, 613-617. doi: 10.1016/j.plantsci.2008. 03.010

Chen, Q., Ma, Y., Yang, Y., Chen, Z., Liao, R., Xie, X., et al. (2013). Genotyping by genome reducing and sequencing for outbred animals. PLoS One 8:e67500. doi: 10.1371/journal.pone.0067500

Comai, L., and Henikoff, S. (2006). TILLING: practical single-nucleotide mutation discovery. Plant J. 45, 684-694. doi: 10.1111/j.1365-313X.2006. 02670.x

Conesa, A., Götz, S., García-Gómez, J. M., Terol, J., Talón, M., and Robles, M. (2005). Blast2GO: a universal tool for annotation, visualization and analysis in functional genomics research. Bioinformatics. 21, 3674-3676. doi: 10.1093/ bioinformatics/bti610

da Câmara Machado, A., Frick, N. S., Kremen, R., Katinger, H., and Laimer da Câmara Machado, M. (1997). "Biotechnological approaches to the improvement of Jatropha curcas," in Proceedings of International Symposium on Jatropha, Managua.

Dhakshanamoorthy, D., Selvaraj, R., and Chidambaram, A. L. A. (2011). Induced mutagenesis in Jatropha curcas L. using gamma rays and detection of DNA polymorphism through RAPD marker. Crit. Rev. Biol. 334, 24-30. doi: 10.1016/ j.crvi.2010.11.004 
Dhillon, R. S., Saharan, R. P., Jattan, M., Rani, T., Sheokand, R. N., Dalal, V., et al. (2014). Molecular characterization of induced mutagenesis through gamma radiation using RAPD markers in Jatropha curcas L. Afr. J. Biotechnol. 13, 806-813. doi: 10.5897/AJB12. 2934

Dierking, E. C., and Bilyeu, K. D. (2009). New sources of soybean seed meal and oil composition traits identified through TILLING. BMC Plant Biol. 9:89. doi: 10.1186/1471-2229-9-89

Doebley, J. F., Gaut, B. S., and Smith, B. D. (2006). The molecular genetics of crop domestication. Cell 127, 1309-1321. doi: 10.1016/j.cell.2006. 12.006

Elshire, R. J., Glaubitz, J. C., Sun, Q., Poland, J. A., Kawamoto, K., Buckler, E. S., et al. (2011). A robust, simple genotyping-by-sequencing (GBS) approach for high diversity species. PLoS One 6:e19379. doi: 10.1371/journal.pone.001 9379

Forster, B. P., Franckowiak, J. D., Lundqvist, U., Thomas, W. T. B., Leader, D., Shaw, P., et al. (2012). "Mutant phenotyping and pre-breeding in barley," in Plant Mutation Breeding and Biotechnology, eds Q. Y. Shu, B. P. Forster, and H. Nakagawa (Wallingford: CABI).

Harloff, H. J., Lemcke, S., Mittasch, J., Frolov, A., Wu, J. G., Dreyer, F., et al. (2012). A mutation screening platform for rapeseed (Brassica napus L.) and the detection of sinapine biosynthesis mutants. Theor. Appl. Genet. 124, 957-969. doi: 10.1007/s00122-011-1760-z

Henry, I. M., Nagalakshmi, U., Lieberman, M. C., Ngo, K. J., Krasileva, K. V., Vasquez-Gross, H., et al. (2014). Efficient genome wide detection and cataloging of EMS-Induced mutations using exome capture and nextgeneration sequencing. Plant Cell 26, 1382-1397. doi: 10.1105/tpc.113.12 1590

Jankowicz-Cieslak, J., Huynh, O. A., Brozynska, M., Nakitandwe, J., and Till, B. J. (2012). Induction, rapid fixation and retention of mutations in vegetatively propagated banana. Plant Biotechnol. J. 10, 1056-1066. doi: 10.1111/j.14677652.2012.00733.x

Jiao, Y., Burke, J., Chopra, R., Burow, G., Chen, J., Wang, B., et al. (2016). A sorghum mutant resource as an efficient platform for gene discovery in grasses. Plant Cell 28, 1551-1562. doi: 10.1105/tpc.16.00373

Jiang, Z., Wang, H., Michal, J. J., Zhou, X., Liu, B., et al. (2016). Genome wide sampling sequencing for SNP genotyping: methods, challenges and future development. Int. J. Biol. Sci. 12, 100-108. doi: 10.7150/ijbs. 13498

King, A., Montes, L., Clarke, J., Affleck, J., Li, Y., Witsenboer, H., et al. (2013). Linkage mapping in the oilseed crop Jatropha curcas L. reveals a locus controlling the biosynthesis of phorbol esters which cause seed toxicity. Plant Biotechnol. J. 11, 986-996. doi: 10.1111/pbi.12092

Liu, H., Bayer, M., Druka, A., Russell, J. R., Hackett, C. A., Poland, J., et al. (2014). An evaluation of genotyping by sequencing (GBS) to map the Breviaristatume (ari-e) locus in cultivated barley. BMC Genomics 15:104. doi: 10.1186/14712164-15-104

Maghuly, F., Bado, S., Jankowicz-Cieslak, J., and Laimer, M. (2017). "Chemical and physical mutagenesis in Jatropha curcas," in Biotechnologies for Plant Mutation Breeding: Protocols, eds J. Jankowicz-Cieslak, T. H. Tai, J. Kumlehn, and B. J. Till (Cham: Springer), 21-38. doi: 10.1007/978-3-319-45021-6_2

Maghuly, F., Haas, B., and Laimer, M. (2016). Biotechnological approaches to growing green energy from Jatropha curcas: challenges due to the undomesticated status of the species. CAB Rev. 11, 1-13. doi: 10.1079/ PAVSNNR201611008

Maghuly, F., Jankowicz-Cieslak, J., Pabinger, S., Till, B. J., and Laimer, M. (2015). Geographic origin is not supported by the genetic variability found in a large living collection of Jatropha curcas with accessions from three continents. Biotechnol. J. 10, 536-551. doi: 10.1002/biot.201400196

Maghuly, F., and Laimer, M. (2013). Jatropha curcas, a biofuel crop: functional genomics for understanding metabolic pathways and genetic improvement. Biotechnol. J. 8, 1172-1182. doi: 10.1002/biot.201300231

Maghuly, F., and Laimer, M. (2017). "Forward and reverse genetics for the improvement of Jatropha," in The Jatropha Genome. Compendium of Plant Genomes, ed. S. Tsuchimoto (Cham: Springer).

Mascher, M., Wu, S. Y., St. Amand, P., Stein, N., and Poland, J. (2013). Application of genotyping-by-sequencing on semiconductor sequencing platforms: a comparison of genetic and reference-based marker ordering in barley. PLoS One 8:e76925. doi: 10.1371/journal.pone.0076925
Mba, C., Afza, R., Bado, S., and Jain, S. M. (2010). "Induced mutagenesis in plants using physical and chemical agents," in Plant Cell Culture: Essential Methods, eds M. R. Davey and P. Anthony (Chichester: Wiley), 111-130.

Minoia, S., Petrozza, A., D’Onofrio, O., Piron, F., Mosca, G., Sozio, G., et al. (2010). A new mutant genetic resource for tomato crop improvement by TILLING technology. BMC Res. Notes. 3:69. doi: 10.1186/1756-05003-69

Mohd-Yusoff, N. F., Ruperao, P., Tomoyoshi, N. E., Edwards, D., Gresshoff, P. M., Biswas, B., et al. (2015). Scanning the effects of ethyl methanesulfonate on the whole genome of Lotus japonicus using second-generation sequencing analysis. G3 5, 559-567. doi: 10.1534/g3.114.014571

Negussie, A., Achten, W. M. J., Verboven, H. A. F., Hermy, M., and Muys, B. (2014). Floral display and effects of natural and artificial pollination on fruiting and seed yield of the tropical biofuel crop Jatropha curcas L. GCB Bioenergy 6, 210-218. doi: $10.1111 / \mathrm{gcbb} .12072$

Ng, P., and Henikoff, S. (2001). Predicting deleterious amino acid substitutions. Genome Res. 11, 863-874. doi: 10.1101/gr.176601

Nielsen, R., Paul, J. S., Albrechtsen, A., and Song, Y. S. (2011). Genotype and SNP calling from next-generation sequencing data. Nat. Rev. Genet. 12, 443-451. doi: $10.1038 / \operatorname{nrg} 2986$

Peterson, B. K., Weber, J. N., Kay, E. H., Fisher, H. S., and Hoekstra, H. E. (2012). Double digest RADseq: an inexpensive method for de novo SNP discovery and genotyping in model and non-model species. PLoS One. 7:e37135. doi: 10.1371/journal.pone. 0037135

Poland, J. A., Brown, P. J., Sorrells, M. E., and Jannink, J.-L. (2012). Development of high-density genetic maps for barley and wheat using a novel two-enzyme genotyping-by-sequencing approach. PLoS One 7:e32253. doi: 10.1371/journal. pone. 0032253

Sato, S., Hirakawa, H., Isobe, S., Fukai, E., Watanabe, A., Kato, M., et al. (2011). Sequence analysis of the genome of an oil-bearing tree, Jatropha curcas L. DNA Res 18, 65-76. doi: 10.1093/dnares/dsq030

Shirasawa, K., Hirakawa, H., Nunome, T., Tabata, S., and Isobe, S. (2016). Genome-wide survey of artificial mutations induced by ethyl methanesulfonate and gamma rays in tomato. Plant Biotechnol. J. 14, 51-60. doi: 10.1111/pbi. 12348

Shu, Q. Y., Forster, B. P., and Nakagawa, H. (2012). "Principles and applications of plant mutation breeding," in Plant Mutation Breeding and Biotechnology, eds Q. Y. Shu, B. P. Forster, and H. Nakagawa (Wallingford: CABI). doi: 10.1079/ 9781780640853.0000

Sidhu, G., Mohan, A., Zheng, P., Dhaliwal, A. K., Main, D., and Gill, K. S. (2015). Sequencing-based high throughput mutation detection in bread wheat. $B M C$ Genomics 16:962. doi: 10.1186/s12864-015-2112-1

Sikora, P., Chawade, A., Larsson, M., Olsson, J., and Olsson, O. (2011). Mutagenesis as a tool in plant genetics, functional genomics, and breeding. Int. J. Plant Genomics 2011:13. doi: 10.1155/2011/314829

Suprasanna, P., and Nakagawa, H. (2012). "Mutation breeding of vegetatively propagated crops," in Plant Mutation Breeding and Biotechnology, eds Q. Y. Shu, B. P. Forster, and H. Nakagawa (Wallingford: CABI).

Till, B. J., Cooper, J., Tai, T. H., Colowit, P., Greene, E. A., Henikoff, S., et al. (2007). Discovery of chemically induced mutations in rice by TILLING. BMC Plant Biol. 7:19. doi: 10.1186/1471-2229-7-19

Tsai, H., Howell, T., Nitcher, R., Missirian, V., Watson, B., Ngo, K. J., et al. (2011). Discovery of rare mutations in populations: TILLING by sequencing. Plant Physiol. 156, 1257-1268. doi: 10.1104/pp.110.169748

Wickland, D. P., Battu, G., Hudson, K. A., DiersMatthew, B. W., and Hudson, E. (2017). A comparison of genotyping-by-sequencing analysis methods on lowcoverage crop datasets shows advantages of a new workflow, GB-eaSy. BMC Bioinformatics 18:586. doi: 10.1186/s12859-017-2000-6

Wu, P., Zhou, C., Cheng, S., Wu, Z., Lu, W., Han, J., et al. (2015). Integrated genome sequence and linkage map of physic nut (Jatropha curcas L.), a biodiesel plant. Plant J. 81, 810-821. doi: 10.1111/tpj.12761

Xin, Z., Li Wang, M., Barkley, N. A., Burow, G., Franks, C., Pederson, G., et al. (2008). Applying genotyping (TILLING) and phenotyping analyses to elucidate gene function in a chemically induced sorghum mutant population. BMC Plant Biol. 8:103. doi: 10.1186/1471-2229-8-103

Yi, C., Zhang, S., Liu, X., Bui, H. T., and Hong, Y. (2010). Does epigenetic polymorphism contribute to phenotypic variances in Jatropha curcas L.? BMC Plant Biol. 10:259. doi: 10.1186/1471-2229-10-259 
Zhang, L., Zhang, C., Wu, P., Chen, Y., Li, M., Jiang, H., et al. (2014). Global analysis of gene expression profiles in physic nut (Jatropha curcas L.) seedlings exposed to salt stress. PLoS One 9:e97878. doi: 10.1371/journal.pone.009 7878

Zhu, Y., Mang, H., Sun, Q., Hipps, A., and Hua, J. (2012). Gene discovery using mutagen-induced polymorphisms and deep sequencing: application to plant disease resistance. Genetics 192, 139-146. doi: 10.1534/genetics.112.14 1986

Zmienko, A., Samelak, A., Kozlowski, P., and Figlerowicz, M. (2014). Copy number polymorphism in plant genomes. Theor. Appl. Genet. 127, 1-18. doi: 10.1007/ s00122-013-2177-7
Conflict of Interest Statement: The authors declare that the research was conducted in the absence of any commercial or financial relationships that could be construed as a potential conflict of interest.

Copyright (c) 2018 Maghuly, Pabinger, Krainer and Laimer. This is an open-access article distributed under the terms of the Creative Commons Attribution License (CC BY). The use, distribution or reproduction in other forums is permitted, provided the original author(s) and the copyright owner are credited and that the original publication in this journal is cited, in accordance with accepted academic practice. No use, distribution or reproduction is permitted which does not comply with these terms. 